\title{
Correction to: A hybrid parallel Harris hawks optimization algorithm for reusable launch vehicle reentry trajectory optimization with no-fly zones
}

\author{
Ya Su${ }^{1} \cdot$ Ying Dai $^{1} \cdot$ Yi Liu ${ }^{1}$
}

Published online: 16 October 2021

(C) Springer-Verlag GmbH Germany, part of Springer Nature 2021

\section{Correction to: Soft Computing}

https://doi.org/10.1007/s00500-021-06039-y

There are some errors in the layout of the paper, the correct ones are as follows:

1. "[1,1]" should be "[-1,1]" (Line 353)

2. " $\boldsymbol{X}(t+1)=\Delta \boldsymbol{X}(t)-E\left|J \boldsymbol{I} \boldsymbol{X}_{\text {rabbit }}(t)-\boldsymbol{X}(t)\right| "$ should be " $\boldsymbol{X}(t+1)=\Delta \boldsymbol{X}(t)-E\left|J \boldsymbol{X}_{\text {rabbit }}(t)-\boldsymbol{X}(t)\right| "$ " (Line 376)

3. " $\boldsymbol{V}_{i}=\boldsymbol{X}_{i}+F\left(\boldsymbol{X}_{\text {best }}-\boldsymbol{X}_{r 1}\right)+F\left(\boldsymbol{X}_{r 2}-\boldsymbol{X}_{r 3}\right)$ " should be " $\boldsymbol{V}_{i}=\boldsymbol{X}_{i}+F\left(\boldsymbol{X}_{\text {best }}-\boldsymbol{X}_{r 1}\right)+F\left(\boldsymbol{X}_{r 2}-\boldsymbol{X}_{r 3}\right)$ " (Line 450)

4. “ $\bar{u}_{k}= \begin{cases}\lambda u_{k-1}+(1-\lambda), & \text { if } \operatorname{sign}\left(u_{k}-u_{k-1}\right) \operatorname{sign}\left(u_{k+1}-u_{k}\right)<0 \\ u_{k}, & \text { else }\end{cases}$

" should be "

$\bar{u}_{k}= \begin{cases}\lambda u_{k-1}+(1-\lambda) u_{k+1}, & \text { if } \operatorname{sign}\left(u_{k}-u_{k-1}\right) \operatorname{sign}\left(u_{k+1}-u_{k}\right)<0 \\ u_{k}, & \text { else }\end{cases}$

" (Line 541)

5. “ $\boldsymbol{X}_{i}(t+1)=$

$\begin{cases}\boldsymbol{X}_{i}(t)+F\left(\boldsymbol{X}_{\text {rabbit }}(t)-\boldsymbol{X}_{r 1}(t)\right)+F\left(\boldsymbol{X}_{r 2}(t)-\boldsymbol{X}_{r 3}(t)\right) & q \geq 0.5 \\ \left(\boldsymbol{X}_{\text {rabbit }}(t)-\boldsymbol{X}_{m}(t)\right)-r_{4}\left(\boldsymbol{L B}+r_{5}(\boldsymbol{U B}-\boldsymbol{L B})\right) & q<0.5\end{cases}$

" should be “"
$\boldsymbol{X}_{i}(t+1)= \begin{cases}\boldsymbol{X}_{i}(t)+F\left(\boldsymbol{X}_{\mathrm{rabbit}}(t)-\boldsymbol{X}_{r 1}(t)\right)+F\left(\boldsymbol{X}_{r 2}(t)-\boldsymbol{X}_{r 3}(t)\right) & q \geq 0.5 \\ \left(\boldsymbol{X}_{\mathrm{rabbit}}(t)-\boldsymbol{X}_{\mathrm{m}}(t)\right)-r_{4}\left(\boldsymbol{L} \boldsymbol{B}+r_{5}(\boldsymbol{U} \boldsymbol{B}-\boldsymbol{L B})\right) & q<0.5\end{cases}$

"(Line 551)

6. " $u=-5$ " should be " $u=-5$ " (Line 661)

7. The last three rows of Tables 3 and 4 should be right aligned.

The correct format is below (Table 4 is the same):

8. "Figs. 8a-c" should be "Figs. 8 (a)-(c)"; "Figs. 8 c" should be "Figs. 8 (c)" (Line 799 and Line 803)

9. “ $\varphi$ " should be “ $\varphi$ " (Line 823, Line 829, Line 895 and Table 5)

10. “ $\left[\dot{Q}_{\max }, q_{\max }, n_{z \max }\right]=\left[200 \mathrm{Btu} / \mathrm{ft}^{2} / \mathrm{s}, 13406 \mathrm{~Pa}\right.$, $\left.2.5 g_{0}\right] "$ should be " $\left[\dot{Q}_{\max }, q_{\max }, n_{z \max }\right]=\left[200 \mathrm{Btu} / \mathrm{ft}^{2} / \mathrm{s}\right.$, $\left.13406 \mathrm{~Pa}, 2.5 \mathrm{~g}_{0}\right]$ " (Lines 869-870)

11. "0.59" should be “-0.59" (Line 922)

12. The figure notes for Figures 10 and 11 should be in black font.

13. "dos Coelho LS" should be "Coelho L dos S" (Line 1075)

14. "Grey Wolf Optimizer" should be "Grey wolf optimizer" (Line 1151)

15. The order of the two references corresponding to lines 1151-1157 is wrong, and the correct format is

Mirjalili S, Mirjalili SM, Hatamlou A (2016) Multiverse optimizer: a nature-inspired algorithm for global optimization. Neural Comput Appl 27:495-513. https://doi. org/10.1007/s00521-015-1870-7

Mirjalili S, Mirjalili SM, Lewis A (2014) Grey wolf optimizer. Adv Eng Softw 69:46-61. https://doi.org/10. 1016/j.advengsoft.2013.12.007

16. The order of the two references corresponding to lines 1207-1214 is wrong, and the correct format is

Zhang DN, Liu Y (2011) RLV reentry trajectory optimization through hybridization of an improved GA and a SQP algorithm. In: AIAA Guidance, Navigation, and Control Conference. https://doi.org/10.2514/6.2011-6658 
Table 3 Comparison of the results of $\mathrm{HHO}$ variants

\begin{tabular}{lllllll}
\hline F & Metric & OPHHODE & PHHCDE & PHHLDE & OPHHCLDE & HPHHO \\
\hline F1 & AVG & $2.3491 \mathrm{e}-313$ & $1.6712 \mathrm{e}-55$ & $9.9909 \mathrm{e}-55$ & $2.4752 \mathrm{e}-125$ & $\mathbf{0 . 0 0 e}+\mathbf{0 0}$ \\
& STD & $\mathbf{0 . 0 0 e + 0 0}$ & $9.1503 \mathrm{e}-55$ & $5.4462 \mathrm{e}-54$ & $1.3556 \mathrm{e}-124$ & $\mathbf{0 . 0 0 e}+\mathbf{0 0}$ \\
F2 & AVG & 0.086026 & 9.4422 & 1.8788 & 0.008728 & $\mathbf{0 . 0 0 0 1 9 1 5 7}$ \\
& STD & 0.1468 & 11.9705 & 6.6536 & 0.017189 & $\mathbf{0 . 0 0 0 3 8 6 5 1}$ \\
F3 & AVG & $\mathbf{8 . 8 8 1 8 e}-\mathbf{1 6}$ & $\mathbf{8 . 8 8 1 8 e}-\mathbf{1 6}$ & $\mathbf{8 . 8 8 1 8 e}-\mathbf{1 6}$ & $\mathbf{8 . 8 8 1 8 e}-\mathbf{1 6}$ & $\mathbf{8 . 8 8 1 8 e}-\mathbf{1 6}$ \\
& STD & $\mathbf{0 . 0 0 e + 0 0}$ & $\mathbf{0 . 0 0 e}+\mathbf{0 0}$ & $\mathbf{0 . 0 0 e}+\mathbf{0 0}$ & $\mathbf{0 . 0 0 e}+\mathbf{0 0}$ & $\mathbf{0 . 0 0 e}+\mathbf{0 0}$ \\
F4 & AVG & $\mathbf{- 1 0 . 1 5 3 2}$ & -5.5612 & -5.994 & $\mathbf{- 1 0 . 1 5 3 2}$ & $-\mathbf{1 0 . 1 5 3 2}$ \\
& STD & 0.0026908 & 1.5568 & 2.1606 & $\mathbf{1 . 2 4 1 1 e}-\mathbf{0 9}$ & $3.6193 \mathrm{e}-07$ \\
+/=/- & 2/2/0 & $3 / 1 / 0$ & $3 / 1 / 0$ & $2 / 2 / 0$ & $\sim$ & \\
ARV & 2.88 & 3.88 & 3.63 & 2.38 & 2.25 & \\
Overall Rank & 3 & 5 & 4 & 2 & 1 & \\
\hline
\end{tabular}

Zhang HP, Wang HL, Li N et al (2020) Time-optimal memetic whale optimization algorithm for hypersonic vehicle reentry trajectory optimization with no-fly zones. Neural Comput Appl 32:2735-2749. https://doi.org/10. 1007/s00521-018-3764-y
The original article has been corrected.

Publisher's Note Springer Nature remains neutral with regard to jurisdictional claims in published maps and institutional affiliations. 